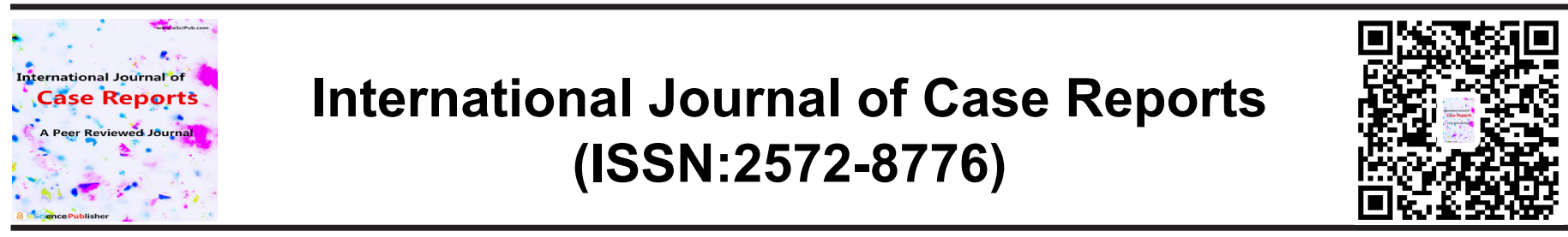

\title{
Prostate stromal tumour: case reports and review of literature
}

\author{
Odusanya Benjamin O.. ${ }^{*}$, Saliu Abdulwaid N. ${ }^{2}$, Oyewole Olugbenga $0 .^{3}$, Mabadeje-Isowo \\ Bilikisu A. ${ }^{4}$, Nwamannah Faith $\mathbf{0 .}^{5}$ \\ ${ }^{1}$ Senior clinical fellow, Urology department, Manchester University NHS Foundation Trust. \\ ${ }^{2}$ Consultant urologist, Department of Surgery, General Hospital, Marina, Lagos. ${ }^{3}$ Consultant \\ Pathologist, Department of Pathology, General Hospital, Marina, Lagos. ${ }^{4}$ Consultant radiation \\ and clinical oncologist Department of Surgery, General Hospital, Marina , Lagos. ${ }^{5}$ House officer, \\ Department of Surgery, General Hospital, Marina, Lagos.
}

\section{ABSTRACT}

\section{Background}

Keywords: Sarcoma; Stromal tum-

Prostate cancer is a common malignancy in men; however, sarcoma of the prostate is an uncommon variant. Sarcoma of the prostate occurs in all age groups with rhabdomyosarcoma being more common in children while leiomyosarcoma occurs more in the older adults. Stromal tumour of uncertain malignant potential (STUMP) occurs in adults. Modalities of treatment include surgery, radiotherapy and chemotherapy used singly or in combination, but all report poor long term survival despite treatment selected. We present 2 cases of prostate stromal tumours.

\section{Case report}

We present two patients in their fourth decade who presented with acute urinary retention. Computed tomography scan was performed in both cases and showed a large tumour arising from the prostate. Prostate specific antigen was less than 4ng/ $\mathrm{ml}$ in the two cases and core needle biopsy performed and samples taken for histology. The histology reports showed embryonal carcinoma and STUMP in the first and second cases respectively.

\section{Conclusion}

Prostate sarcomas are uncommon but may pursue aggressive clinical course while STUMP has a variable course. Early diagnosis and use of combined modalities of treatment may be required to improve survival.

our; Prostate cancer; STUMP

*Correspondence to Author:

Odusanya Benjamin O.

Senior clinical fellow, Urology department,Manchester University NHS Foundation Trust.

How to cite this article:

Odusanya Benjamin O., Saliu Abdulwaid N., Oyewole Olugbenga O., Mabadeje-Isowo Bilikisu A., Nwamannah Faith O.. Prostate stromal tumour: case reports and review of literature. International Journal of Case Reports, 2021; 5:215.

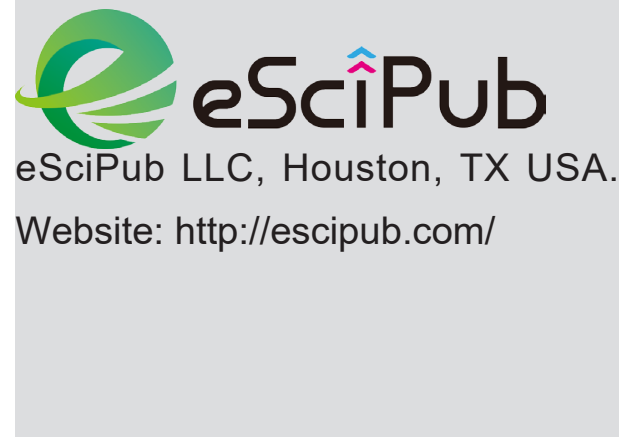




\section{Introduction}

Prostate malignancy is one of the commonest malignancies in men. It is the leading cause of cancer death in men. Majority of these are adenocarcinoma with sarcoma being uncommon. Sarcoma of the prostate account for less than $0.1 \%$ of prostate malignancies. ${ }^{[1,2]}$ Whilst leiomyoma is more common in adults, rhabdomyosarcoma is prevalent in the paediatric age group. ${ }^{[3]}{ }^{[4]}$ Specialised stromal tumour of the prostate sarcoma include stromal tumour of uncertain malignant potential(STUMP) and stromal sarcoma. ${ }^{[5]}$ Prostate stromal proliferation of uncertain malignant potential was described in 1998 by Gaudin et al. ${ }^{[6]}$ The diagnosis is usually with transrectal ultrasound guided biopsy or transurethral resection of the prostate. Various modalities of treatment have been attempted in the treatment of prostate sarcoma but all modalities of treatment have a poor prognosis. [7] Because of the rarity of these tumours, we report two cases of stromal tumours of the prostate in young adults.

\section{Case report}

31 year old presented via the emergency room with a day history of inability to pass urine. This was relieved by urethral catheterisation. He had a preceding history of urethral discharge, high grade fever and both storage and voiding lower urinary tract symptoms. He admitted to a history of multiple sexual partners. There was no history of haematuria, previous instrumentation or catheterisation or trauma. Initial digital rectal examination revealed a grossly enlarged prostate that was warm boggy and tender. He was initially treated for acute bacterial prostatitis and initial trial without catheter was successful. He was treated with antibiotic and his fever subsided.

Subsequently, he re-presented within two weeks with recurrent acute urinary retention and fever had re-occurred. An assessment of prostatic abscess was made which was confirmed on transrectal ultrasound. An attempt at transrectal ultrasound guided drainage of abscess yielded scanty amount of pus. His clinical condition deteriorated and abdominal ultrasound done showed intra-abdominal abscess for which attempt was made to drain percutaneously with no success. He underwent laparotomy and intra-operatively, no abscess was detected, only blood clots and a pelvic mass. He was noted to be bleeding excessively at surgery. With the suspicion of a coagulopathy, surgery was concluded expeditiously with just lavage and a drain left in situ. Laboratory investigation post-operatively confirmed a coagulopathy (International normalised ratio [INR] of 1.9) which was corrected with administration of fresh frozen plasma. INR was repeated and revealed INR of 1.13.

As the patient's condition continued to deteriorate, he developed a perineal swelling which was warm and tender; a palpable suprapubic swelling was also noticed. In addition, rectal pain, tenesmus, intermittent rectal bleeding and discharge were observed. The patient had persistent pain and gross swelling in left lower limb. X-ray of the left thigh showed no bony abnormality, only soft tissue swelling. A computed tomography was requested which show ed a heterogeneous mass with minimal enhancement arising from the pelvis with areas low attenuation showing cystic necrosis. Abscess collection within the mass seen as hypodense collection with rim enhancement measuring 243 milliliters. The mass displaced the bladder cephalad and compressed the rectum and sigmoid colon. No para-aortic lymphadenopathy, ascites, liver,bone or lung metastasis was seen. A diagnosis of prostatic sarcoma was made at this stage. Prostate Specific Antigen level was $2.35 \mathrm{ng} / \mathrm{ml}$.

$\mathrm{He}$ later developed features of intestinal obstruction as a result of extrinsic compression by the mass. He had emergency transverse loop colostomy and a trucut biopsy of the pelvic mass was taken for histology at this surgery. Histopathology confirmed embryonal rhabdomyosarcoma of the prostate. At this stage, the patient had developed bilateral hydronephrosis on abdominal ultrasound and biochemical 
investigations showed renal impairment with eGFR of $30 \mathrm{mls} / \mathrm{min} / 1.73 \mathrm{~m}^{2}$. Oncology consult was sent but patient was deemed unfit for chemotherapy or radiotherapy as his clinical condition has deteriorated and his performance status was four. He later developed bilateral deep vein thrombosis of both lower limbs which were confirmed on Doppler for which he had enoxaparin for anticoagulation.

Eventually, the patient succumbed to his illness.

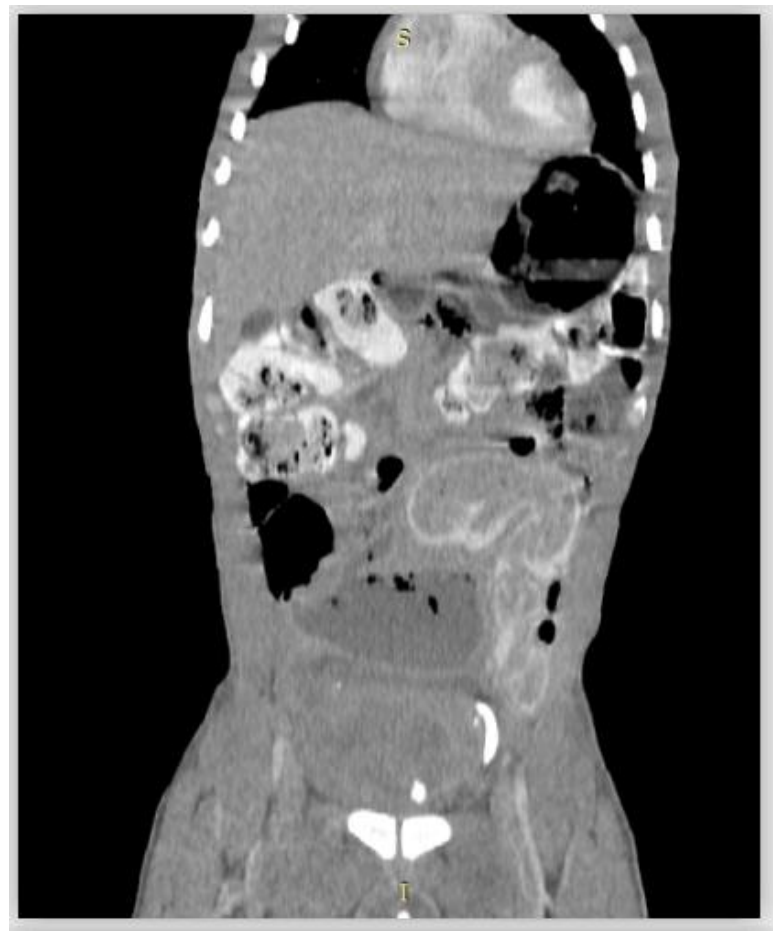

Pic 1 coronal reconstruction

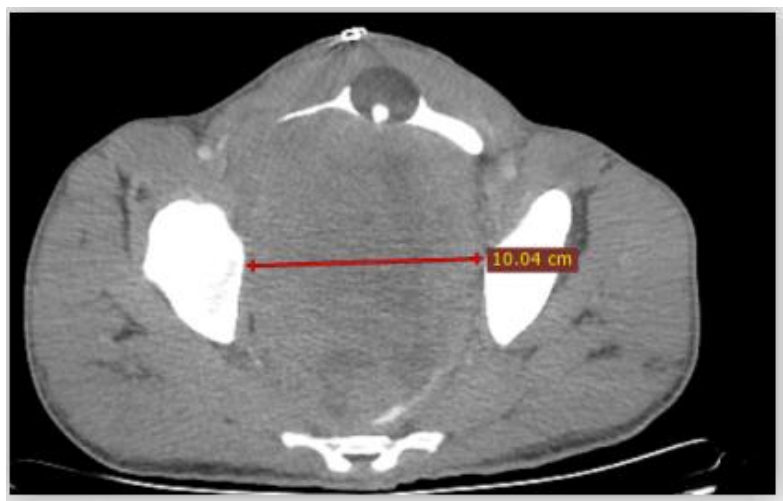

Pic 2 axial image with measurement

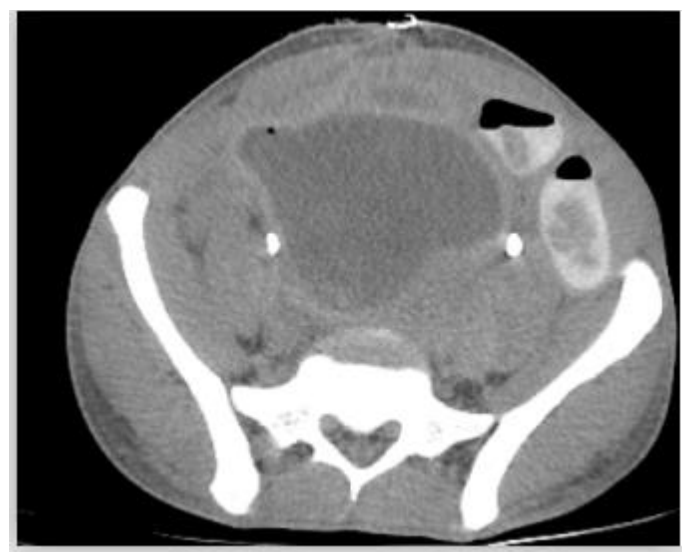

Pic 3 axial showing necrotic area within the tumour 
Odusanya Benjamin O. et al., IJCR, 2021; 5:215

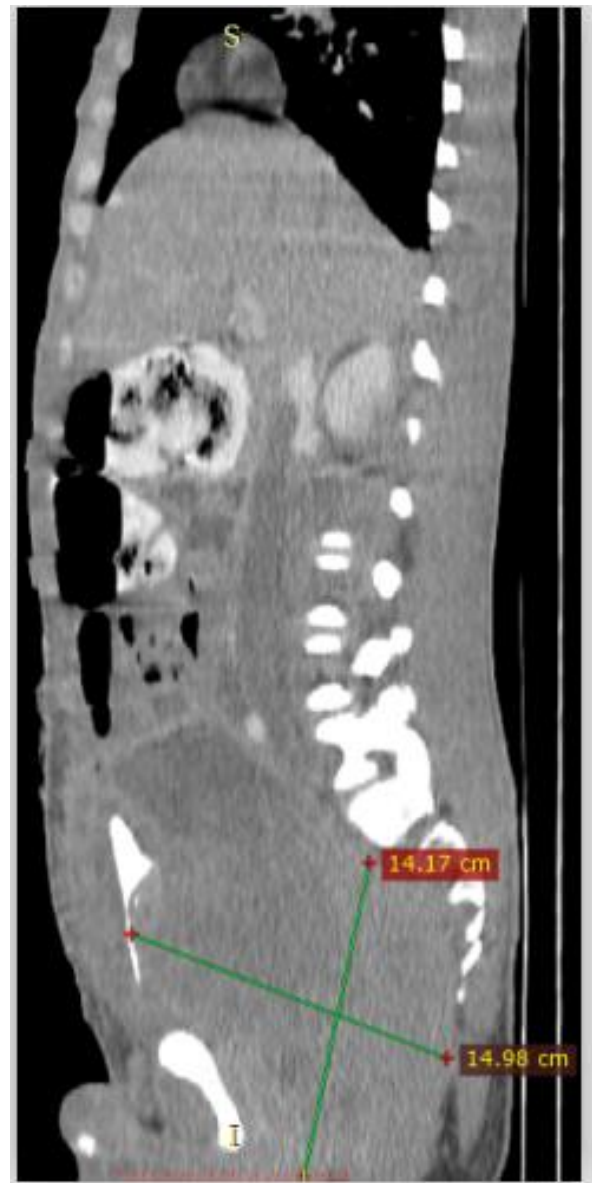

Pic 4 saggital reconstruction with measurement

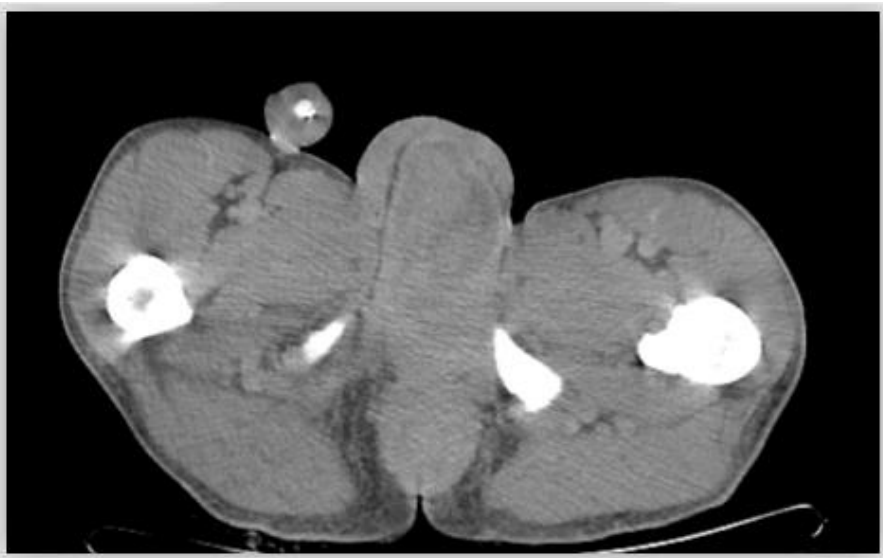

Pic 5 axial image showing perineal extension
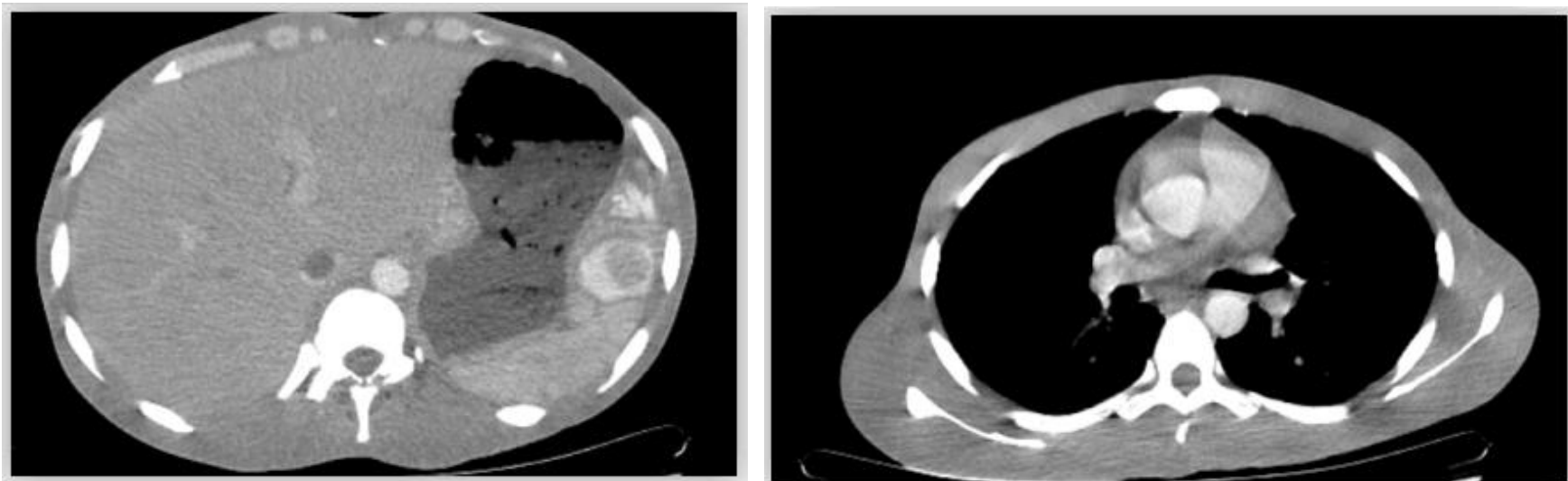

Pic 6 and 7 no liver or lung metastasis

IJCR: https://escipub.com/international-journal-of-case-reports/ 


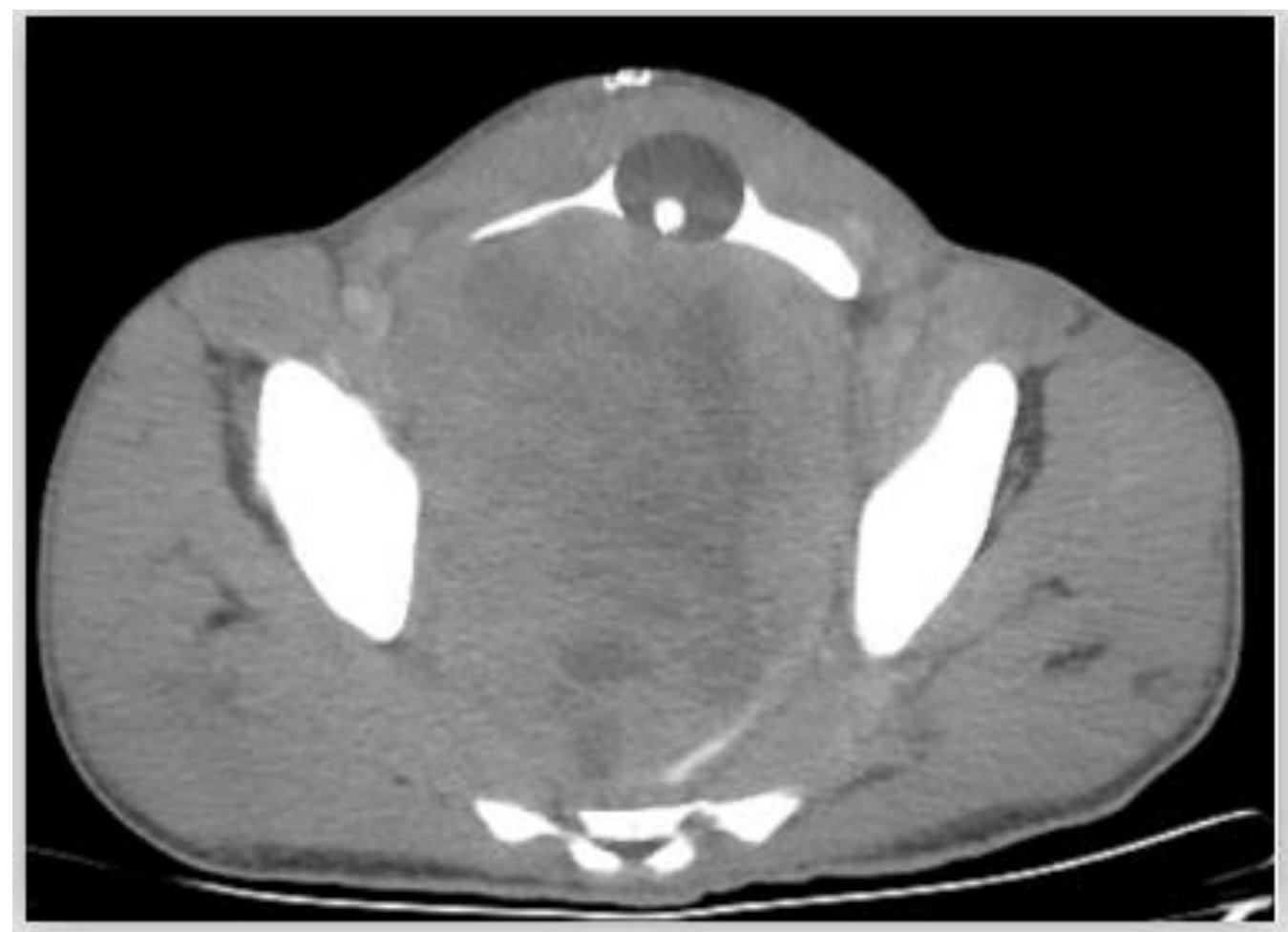

Pic 8 axial image showing catheter balloon in the bladder

\section{Case 2}

30 years old with a day history of inability to pass urine and was relieved by urethral catheterisation. He had a 3 months history of progressive voiding lower urinary tract symptoms but he denied storage symptoms. He has no history of haematuria, dysuria, urethral discharge, prior urethral instrumentation or trauma. There was no history suggestive of renal impairment. There is a history of weight loss despite having good appetite. He has tenesmus and constipation which alternates with watery stool but no haematochezia, perineal pain or swelling. He admits to moderate aching waist pain which radiates to right lower limb but there is no paraesthesia or weakness. He also has swelling of both lower limbs of recent onset. No history of fever, cough, jaundice, headache or seizures.

At onset of symptoms, he used herbal remedy. He takes alcohol and smokes marijuana but does not take tobacco. Relevant examination findings include a suprapubic mass extending to just below the umbilicus which was firm and fixed, immobile and was present even when a urethral catheter was in-situ draining clear urine. Digital rectal examination revealed an enlarged prostate almost occluding the rectal lumen, it was firm, smooth with no nodules and rectal mucosa was mobile with no separate rectal masses felt. A working diagnosis of prostate sarcoma was made.

Apart from a packed cell volume of 36 per cent, his full blood count and renal function test, liver function test and clotting profile were essentially normal. His prostate specific antigen test was $3.13 \mathrm{ng} / \mathrm{ml}$. Initially he had an abdomino-pelvic ultrasound and later an abdominopelvic and chest computed tomography scan which revealed grossly enlarged prostate measuring $1211 \mathrm{~g}$ with multiple necrotic areas. There was bilateral hydroureter and hydronephrosis but no liver, lung or bony metastasis seen. He had transrectal ultrasound guided prostate biopsy and initial histopathology suggested prostate sarcoma. Additionally, immunohistochemistry showed the tumour was vimentin positive but negative for S-100, CD45,P63, progesterone, cytokeratin (AE1/AE3). Final pathology was prostate stromal proliferation of uncertain malignant potential. He was sent to the oncologist for radiation and chemotherapy but patient declined further treatment and self-discharged. 


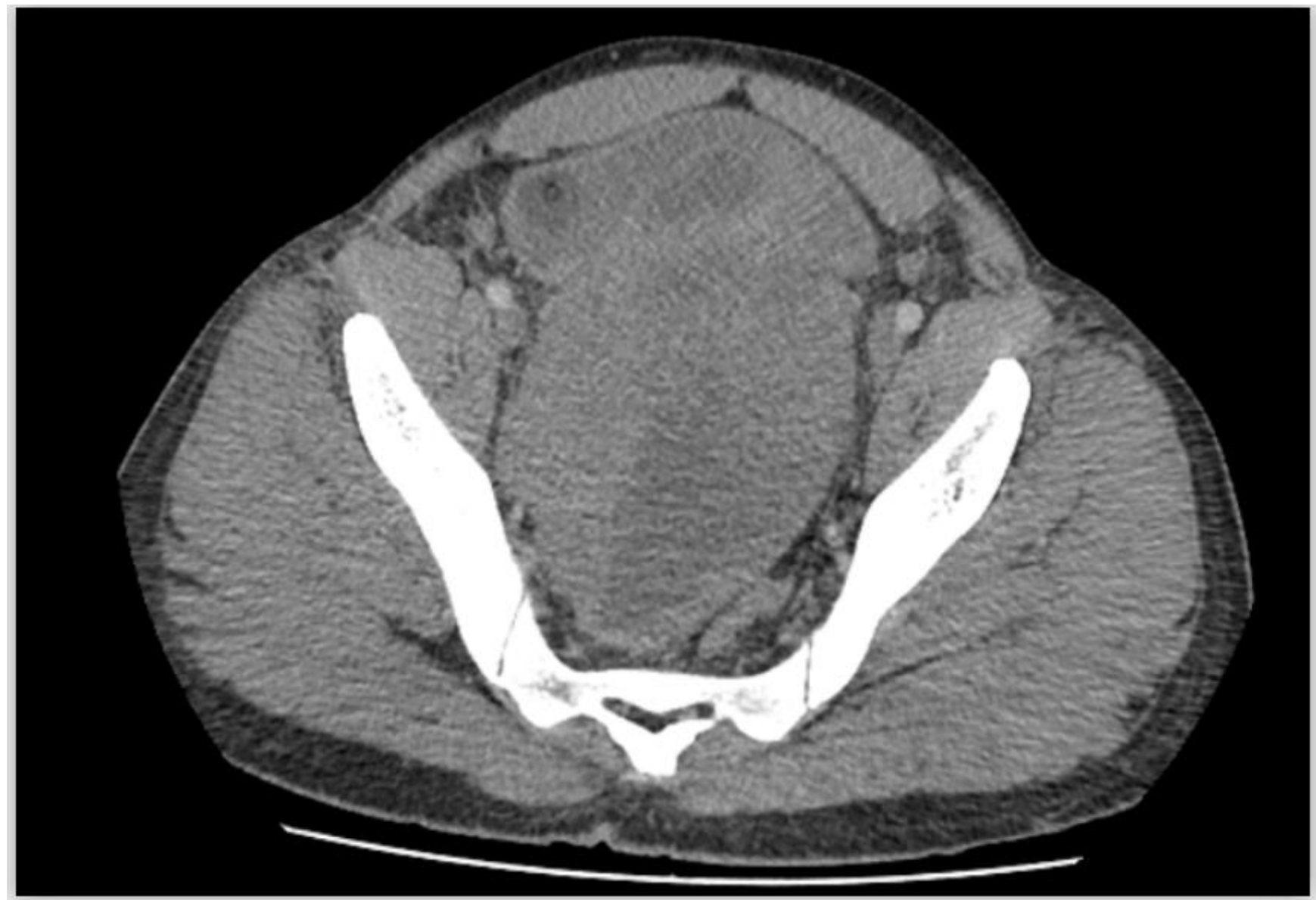

Pic 1 axial pre-contrast

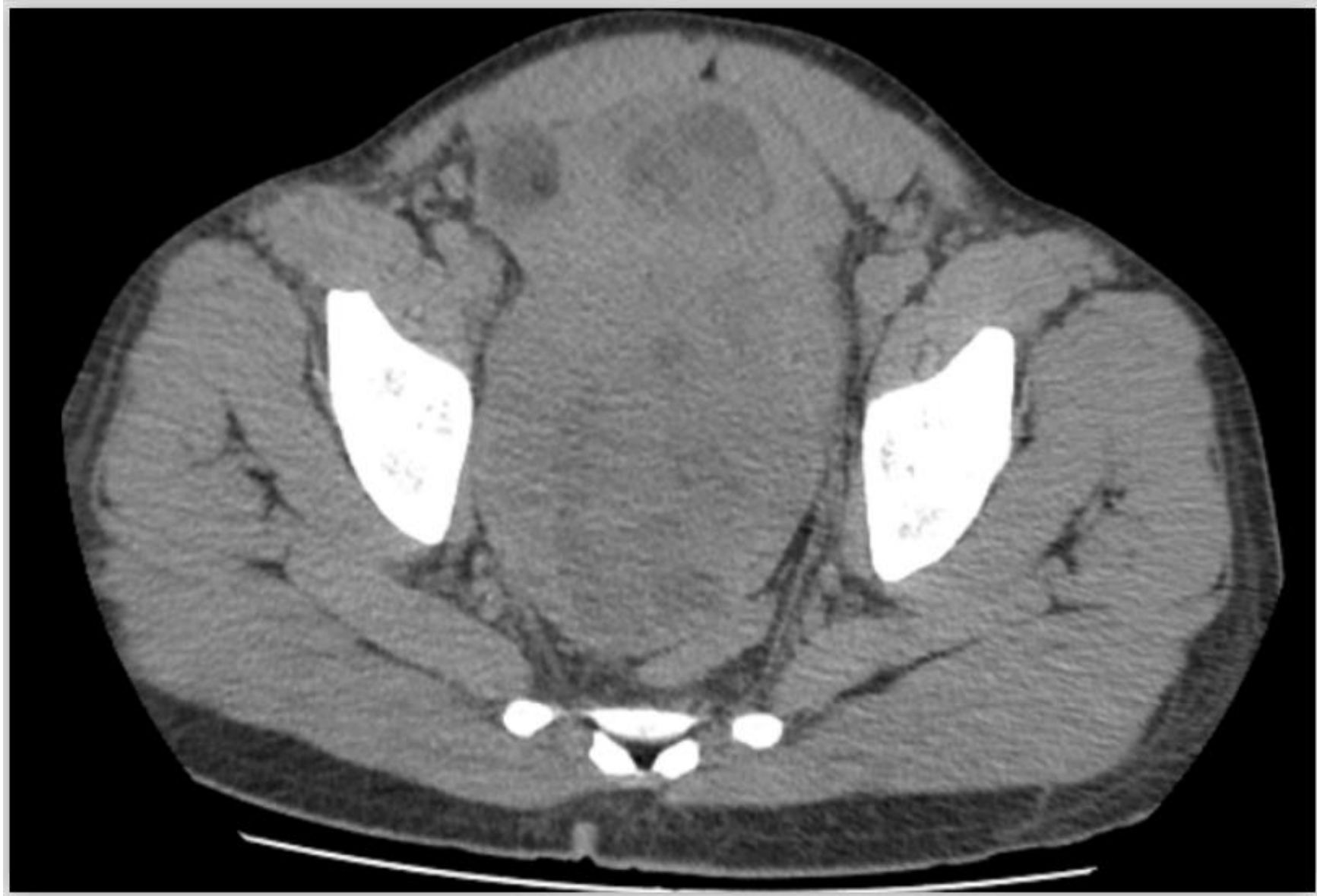

Pic 2 axial post contrast 
Odusanya Benjamin O. et al., IJCR, 2021; 5:215
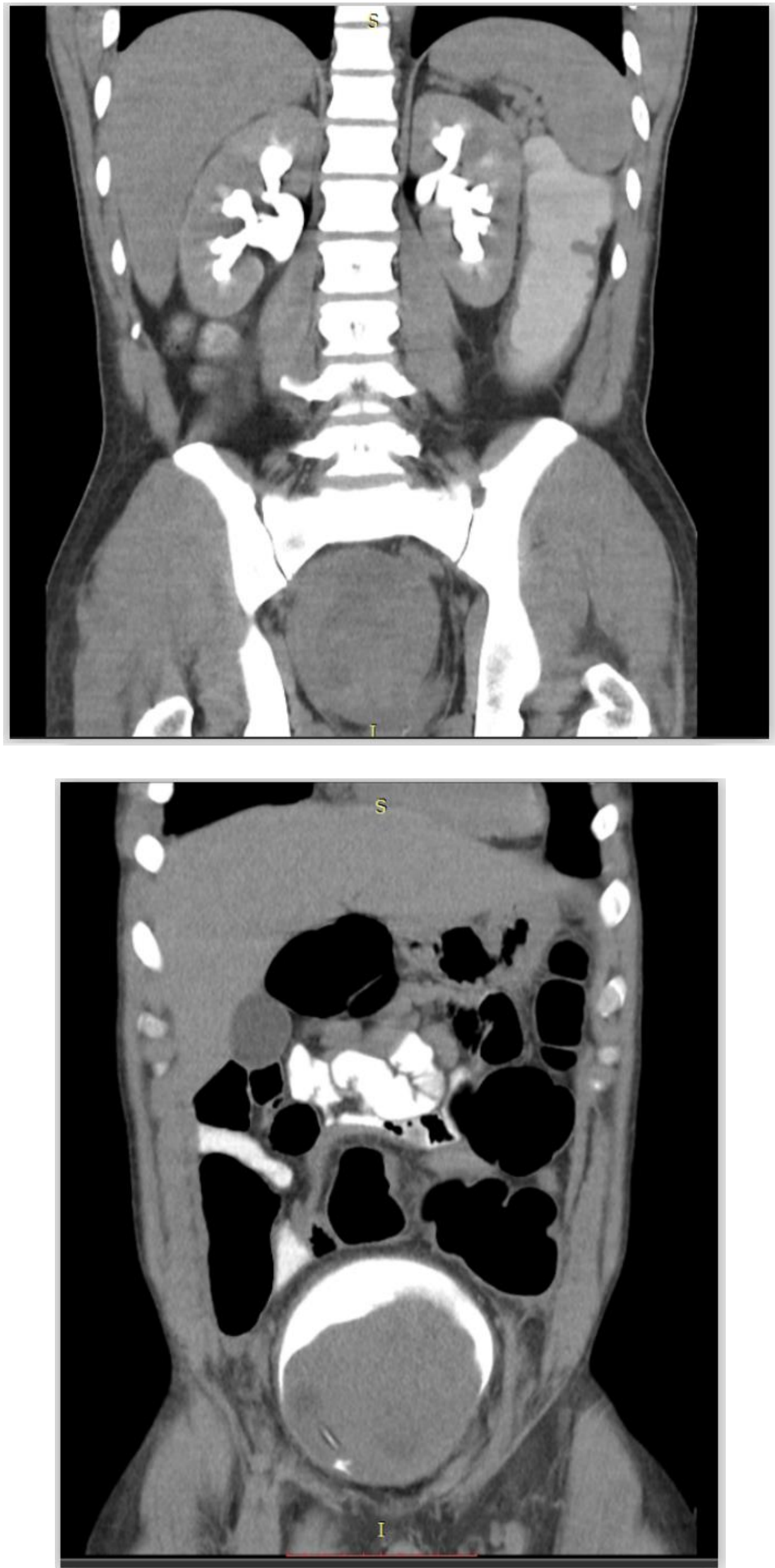

Pic 3 and 4 coronal images with contrast in kidney and bladder respectively IJCR: https://escipub.com/international-journal-of-case-reports/ 
Odusanya Benjamin O. et al., IJCR, 2021; 5:215

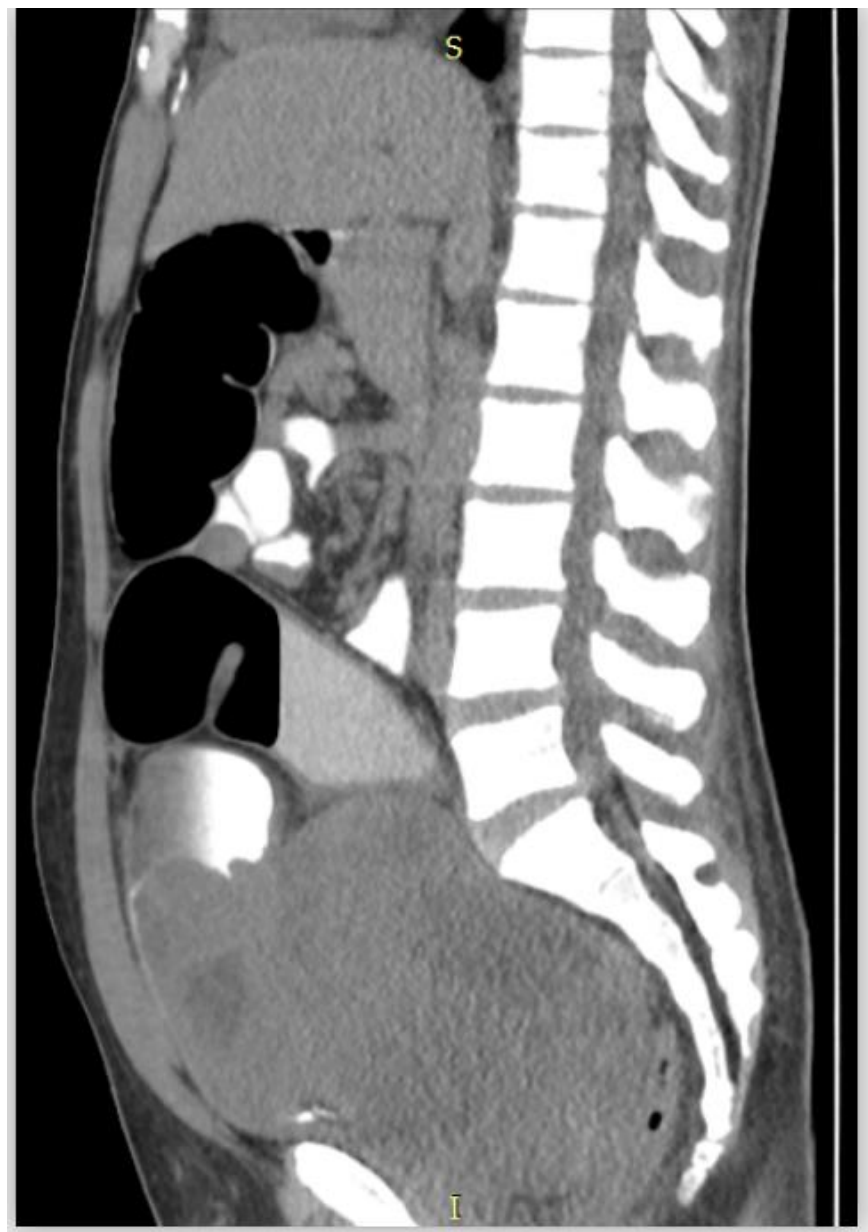

Pic 5 saggital reconstruction showing relationship to bladder

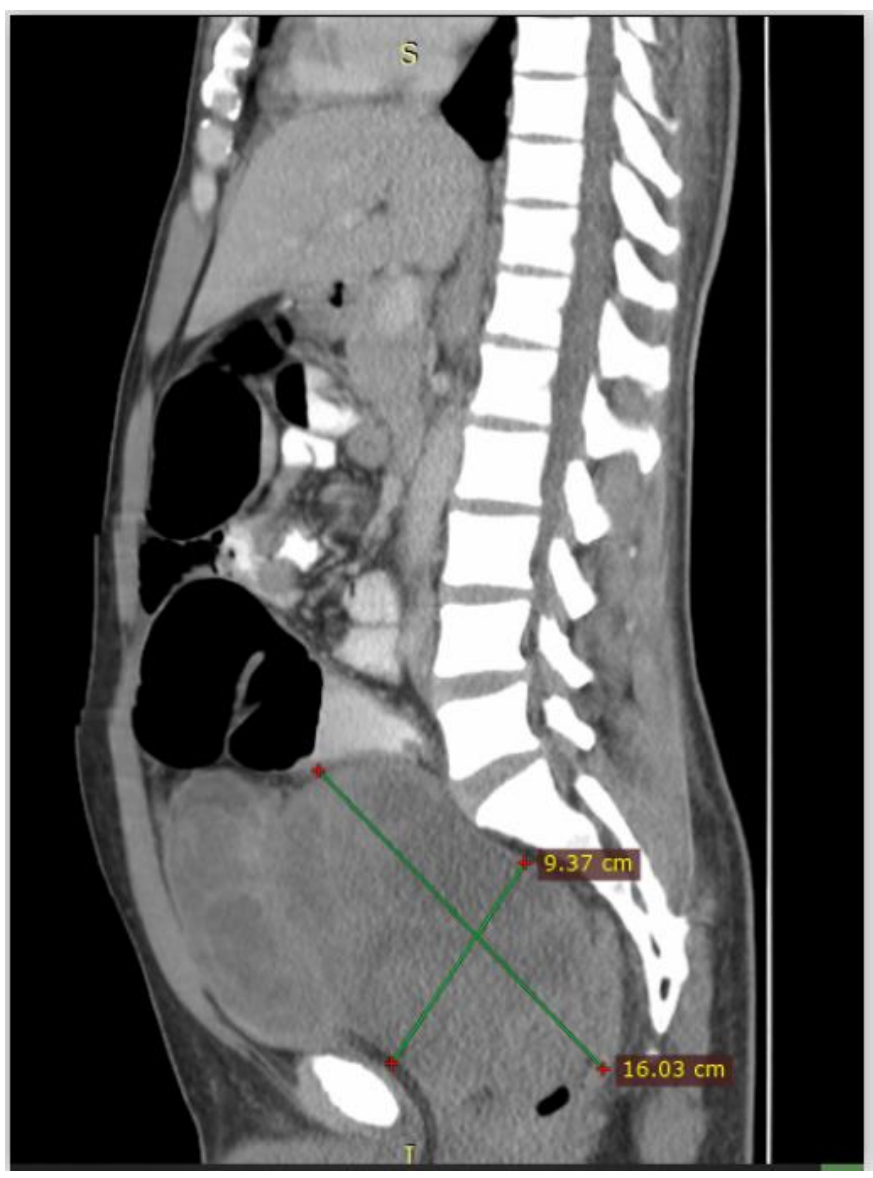

Pic 6 saggital reconstruction with measurement 

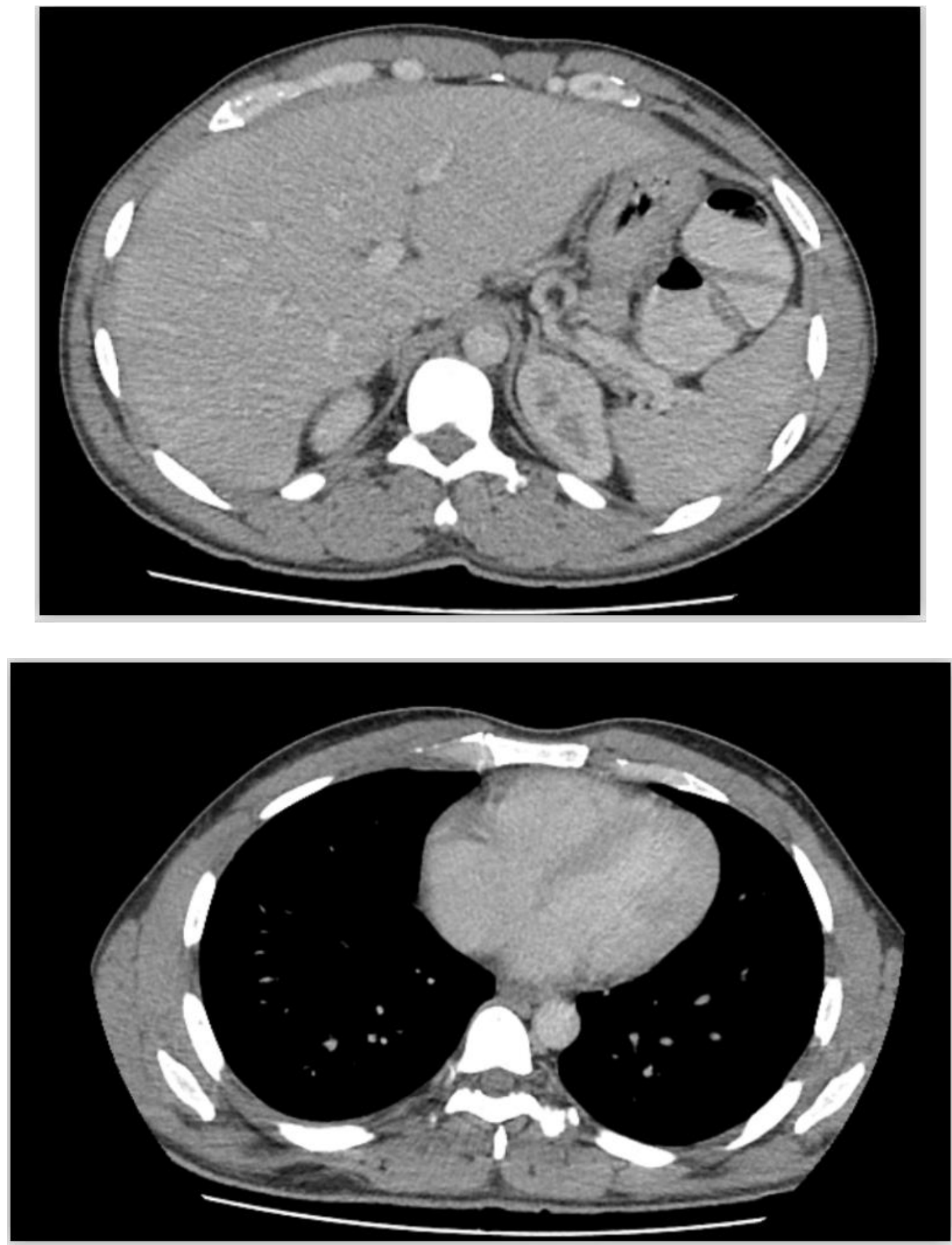

Pic 7 and 8 No metastasis in liver and lungs

\section{Discussion}

Prostate sarcoma is a rare disease and thus the number needed for randomised trial to provide high quality of evidence are uncommon even in this era of evidence based medicine. A study [8] in the United States reported 38 cases over a 30 year period while another study ${ }^{[7]}$ reported only 25 cases over a 20 year period. In parti- cular for embryonal sarcoma of the prostate, as recently as 1992, Waring et al reported only 9 cases in literature. [9] By 2010, reported cases of embryonal sarcoma had grown to 25 cases. [10]
Prostate sarcoma and STUMP are frequently reported together. Two large case series reported 22 and 50 cases respectively. ${ }^{[5,6] ~ H e r a w i ~}$ et al described seven cases of STUMP in their series which were associated with sarcoma occurring concurrently or subsequently. ${ }^{[5]}$

The median age of presentation by most authors for prostate sarcoma was less than 50 years while for STUMP it was greater than 50 years. ${ }^{4-}$ $6,10,11]$ Our patients developed symptoms at 30 and 31 years for the first and second case respectively which was younger than the 
reported median age in literature. A study [7] reported a median age of 37 years with a range of 18 to 81 years while another study [4] reported a median age of 49 years.

Majority of patients with prostate stromal tumour present with symptoms of urinary obstruction. This is because of the often large tumour size at diagnosis. ${ }^{[4,6,11]}$ Other symptoms include pelvic or perineal pain, rectal pain, constipation, haematuria and weight loss. [4, 7] The cases presented had the above symptoms and in addition one had fever initially and later developed perineal swelling, a symptom reported in historical reports. [12] Examination finding often reveal an enlarged firm and smooth prostate in contrast to hard nodular prostate found in carcinoma. Advanced cases such as the both cases we had may present with a suprapubic mass which may reach up to the umbilicus even after catheter has emptied the bladder. [9, 12] Thus rapid development of symptoms of bladder neck obstruction, with rectal or perineal pain with associated weight loss in a young patient should be viewed with high index of suspicion for prostate sarcoma.

Ultrasound guided needle biopsy is a common method of diagnosis. Other methods include transurethral resection and transperineal biopsy. One of our patients had transrectal ultrasound biopsy while the other had needle biopsy was performed intra-operatively at a surgery for palliation. Prostate specific antigen level is usually normal due to the non-epithelial origin of these tumours. ${ }^{4,11]}$

Imaging modalities include ultrasound and three dimensional imaging such as computed tomography (CT) scan and magnetic resonance imaging. CT scan shows large pelvic masses with heterogeneous enhancement and heterogeneous attenuation showing areas of cystic degeneration and necrosis. The limitation of CT being difficulty to differentiate between tumours arising from the prostate and those arising from the bladder base. ${ }^{[13]}$

Magnetic resonance imaging is thought to better delineate soft tissue tumours. It is useful in determining the organ of origin, local extension, presence of lymphadenopathy and surgical planning. On T1-weighted image, most prostate sarcoma appeared as homogenous low signal masses while on T2-weighted images, they are invariably heterogeneous with intermediate and high T2 signals. Majority show heterogeneous contrast enhancement and impeded diffusion on diffusion weighted imaging reflecting their high cellularity. $[13,14]$ The patients presented had normal prostate specific antigen level and abdominopelvic CT scan was done in both cases .

Nodal involvement on imaging is common in rhabdomyosarcoma but is uncommon in leiomyosarcoma. ${ }^{[9,13]}$ Both leiomyosarcoma and rhabdomyosarcoma metastasize to the lung as a primary site. Rhabdomyosarcoma metastasize to bone preferentially whereas leiomyosarcoma spreads to the liver. $[13,15,16]$ Unlike prostate carcinoma which produces osteoblastic bony metastasis in the pelvis and spine predominantly, rhabdomyosarcoma causes osteolytic lesions in the bone with a more diffuse distribution. [9]

Various histological types of prostate sarcoma exist which include leiomyosarcoma, rhabdomyosarcoma and carcinosarcoma. [4, 11] Variants of rhabdomyosarcoma include pleiomorphic, alveolar and embryonal subtypes. $\left.{ }^{[9,} 15\right]$. Four histologic subtypes of STUMP which include degenerative atypia, hypercellular, mixed and phyllodes type have been described. [5] Although distinguishing prostate sarcoma from STUMP is difficult, the degree of cellularity, mitotic activity, necrosis, and extension into surrounding organs are some of the criteria used. [17]

Surgery, radiotherapy and chemotherapy have been used singly or in various combinations in the treatment of prostate sarcoma. Transurethral resection of the prostate may be performed and may be diagnostic for patients who present with acute urinary retention. Some authors advocate radical cystoprostatectomy with negative margins as the main treatment for 
non-metastatic disease. [7] However, others suggest a combination of surgery with chemotherapy and radiotherapy. These authors propose a survival advantage for this multimodal treatment. $[4,11,18]$ In a contemporary series, De Bari et al ${ }^{[18]}$ reported an improved local control and overall survival in patients treated with a combination of surgery and radiotherapy.

Chemotherapeutic agents used include vincristine, cyclophosphamide, adriamycin, ifosfamide. Radiotherapy dose ranged from 40-60 Grays. ${ }^{[11,19]}$ Konno et al ${ }^{[20]}$ reported cure of a case with combination of long course of chemotherapy combined with radiotherapy.

Unlike children, rhabdomyosarcoma has a poor prognosis in adult, inspite of recent advances in treatment. A study ${ }^{[8]}$ from Sloan Kettering Center reported a worse cancer specific survival and overall survival for rhabdomyo- sarcoma compared to leiomyosarcoma. Wang et al ${ }^{[7]}$ in their series reported $76 \%$ dead at 21 months and a five year survival of $11.3 \%$. Specifically for adult embryonal sarcoma, Waring et al [9] reported a median survival of only 8 months which is similar to the findings of Bisceglia et al. [10] One of our patients succumb- ed to the disease in 4 months. In a multivariate analysis, Dotan et al ${ }^{[21]}$ found large tumor size and presence of metastasis as poor prognostic factor. Both patients we managed presented with large tumor size.

The limitation of this report is that none of the patients managed received definitive treatment and follow-up was not possible.

\section{Conclusion}

Prostate sarcoma is an aggressive malignancy with rapid progression and high mortality rate in adults. Early diagnosis and treatment is required for a favourable outcome.

\section{References}

[1] Tetu B, Srigley JR, Bostwick DG. Soft tissue tumours. In: Bostwick DG, editor. Pathology of the prostate. New York: Churchill Livingstone; 1990. p. 117.

[2] Varghese SL, Grossfeld GD. The prostatic gland: malignancies other than adenocarcino- mas.
Radiologic Clinics of North America. 2000; 38(1):179-202.

[3] Morse M, Whitmore W. Genitourinary sarcomas in adults. Surgical Management of Soft Tissue Sarcomas Philadelphia, PA: Lea and Febiger. 1989:189-207.

[4] Sexton WJ, Lance RE, Reyes AO, Pisters PW, Tu S-M, Pisters LL. Adult prostate sarcoma: the MD Anderson Cancer Center experience. The Journal of Urology. 2001;166(2):521-5.

[5] Herawi M, Epstein J I. Specialized stromal tumors of the prostate: a clinicopathologic study of 50 cases. Am J Surg Pathol 2006;30(6): 694704.

[6] Gaudin P B, Rosai J, Epstein J I. Sarcomas and related proliferative lesions of specialized prostatic stroma: a clinicopathologic study of 22 cases. Am J Surg Pathol 1998;22(2):148-62.

[7] Wang X, Liu L, Tang H, Rao Z, Zhan W, Li X, et al. Twenty-five cases of adult prostate sarcoma treated at a high-volume institution from 1989 to 2009. Urology. 2013;82(1):160-5.

[8] Musser JE, Assel M, Mashni JW, Sjoberg DD, Russo P. Adult prostate sarcoma: the Memorial Sloan Kettering experience. Urology. 2014;84 (3):624-8.

[9] Waring PM, Newland RC. Prostatic embryonal rhabdomyosarcoma in adults. A clinicopathologic review. Cancer. 1992;69(3):755-62.

[10] Bisceglia M, Magro G, Carosi I, Cannazza V, Ben Dor D. Primary embryonal rhabdomyosarcoma of the prostate in adults: report of a case and review of the literature. International Journal of Surgical Pathology. 2011;19(6): 8317.

[11] Janet NL, May A-W, Akins RS. Sarcoma of the prostate: a single institutional review. American Journal of Clinical Oncology. 2009;32(1):27-9.

[12] Melicow M, Pelton T, Fish G. Sarcoma of the prostate gland: review of literature; table of classification; report of four cases. The Journal of Urology. 1943;49(5):675-707.

[13] Andreou A, Whitten C, MacVicar D, Fisher C, Sohaib A. Imaging appearance of sarcomas of the prostate. Cancer Imaging. 2013;13(2):228.

[14] Chang JM, Lee HJ, Lee S, Byun S-S, Choe G, $\mathrm{Kim} S$, et al. Unusual tumours involving the prostate: radiological-pathological findings. The British Journal of Radiology. 2008;81(971): 90715.

[15] Agrons GA, Wagner BJ, Lonergan GJ, Dickey GE, Kaufman MS. From the archives of the AFIP. Genitourinary rhabdomyosarcoma in children: radiologic-pathologic correlation. Radiographics. 1997;17(4):919-37. 
[16] Vandoros GP, Manolidis T, Karamouzis MV, Gkermpesi M, Lambropoulou M, Papatsoris AG, et al. Leiomyosarcoma of the prostate: case report and review of 54 previously published cases. Sarcoma. 2008;2008.

[17] De Berardinis E, Busetto GM, Antonini G, Giovannone R, Di Placido M, Maglioccia FM, et al. Incidental prostatic Stromal Tumor of Uncertain Malignant Potential (STUMP): Histopathological and immunohistochemical findings. Urologia Journal. 2012;79(1):65- .

[18] De Bari B, Stish B, Ball MW, Habboush Y, Sargos $P$, Krengli $M$, et al. Adult prostatic sarcoma: a contemporary multicenter Rare Cancer Network study. The Prostate. 2017; 77 (10):1160-6.
[19] Ciammella P, Galeandro M, D'Abbiero N, Palmieri T, Donini E, lotti C. Prostate embryonal rhabdomyosarcoma in adults: Case report and review of literature. Reports of Practical Oncology \& Radiotherapy. 2013;18(5):310-5.

[20] Konno M, Mitsuzuka K, Yamada S, Yamashita S, Kaiho $Y$, Ito $A$, et al. A case of adult metastatic rhabdomyosarcoma of the prostate cured by long-term chemotherapy with local radiation. Urologia Internationalis. 2019;102(1):118-21.

[21] Dotan ZA, Tal R, Golijanin D, Snyder ME, Antonescu C, Brennan MF, et al. Adult genitourinary sarcoma: the 25-year Memorial Sloan-Kettering experience. The Journal of Urology. 2006;176(5):2033-9. 